ANNALES

UNIVERSITATIS MARIAE CURIE-SKŁODOWSKA

LUBLIN - POLONIA

\title{
On inclusion relationships of certain subclasses of meromorphic functions involving integral operator
}

ABSTRACT. In this paper, we introduce some subclasses of meromorphic functions in the punctured unit disc. Several inclusion relationships and some other interesting properties of these classes are discussed.

1. Introduction. Let $\mathcal{M}$ denote the class of functions $f(z)$ of the form

$$
f(z)=\frac{1}{z}+\sum_{k=0}^{\infty} a_{k} z^{k},
$$

which are analytic in the punctured open unit disc

$$
E^{*}=\{z: z \in \mathbb{C} \text { and } 0<|z|<1\}=E \backslash\{0\} .
$$

If $f(z)$ is given by $(1.1)$ and $g(z)$ is given by

$$
g(z)=\frac{1}{z}+\sum_{k=0}^{\infty} b_{k} z^{k}
$$

2000 Mathematics Subject Classification. 30C45, 30C50.

Key words and phrases. Meromorphic functions, functions with bounded boundary and bounded radius rotation, quasi-convex functions, close-to-convex functions, generalized hypergeometric functions, functions with positive real part, Hadamard product (or convolution), linear operators. 
we define the Hadamard product (or convolution) of $f(z)$ and $g(z)$ by

$$
(f \star g)(z)=\frac{1}{z}+\sum_{k=0}^{\infty} a_{k} b_{k} z^{k}=(g \star f)(z) \quad(z \in E) .
$$

Let $P_{k}(\rho)$ be the class of functions $p(z)$ analytic in $E$ with $p(0)=1$ and

$$
\int_{0}^{2 \pi}\left|\frac{\operatorname{Re} p(z)-\rho}{1-\rho}\right| d \theta \leq k \pi, z=r e^{i \theta},
$$

where $k \geqslant 2$ and $0 \leq \rho<1$. This class was introduced by Padmanbhan et al. in [16]. We note that $P_{k}(0)=P_{k}$, see [17], $P_{2}(\rho)=P(\rho)$, the class of analytic functions with positive real part greater than $\rho$ and $P_{2}(0)=P$, the class of functions with positive real part. From (1.4) we can easily deduce that $p(z) \in P_{k}(\rho)$ if and only if, there exists $p_{1}(z), p_{2}(z) \in P(\rho)$ such that for $z \in E$,

$$
p(z)=\left(\frac{k}{4}+\frac{1}{2}\right) p_{1}(z)-\left(\frac{k}{4}-\frac{1}{2}\right) p_{2}(z) .
$$

In recent years, several families of integral operators and differential operators were introduced using Hadamard product (or convolution). For example, we choose to mention the Rushcheweyh derivative [18], the CarlsonShaffer operator [1], the Dziok-Srivastava operator [4], the Noor integral operator [14], also see $[3,5,6,11]$. Motivated by the work of N. E. Cho and K. I. Noor $[2,9]$, we introduce a family of integral operators defined on the space of meromorphic functions in the class $\mathcal{M}$. By using these integral operators, we define several subclasses of meromorphic functions and investigate various inclusion relationships and some other properties for the meromorphic function classes introduced here.

For complex parameters $\alpha_{1}, \ldots, \alpha_{q}$ and $\beta_{1}, \ldots, \beta_{s}\left(\beta_{j} \in \mathbb{C} \backslash \mathbb{Z}_{0}^{-}, j=1, \ldots, s\right.$; $\left.\mathbb{Z}_{0}^{-}=\{0,-1,-2, \ldots\}\right)$ we now define the function $\phi\left(\alpha_{1}, \ldots, \alpha_{q} ; \beta_{1}, \ldots, \beta_{s} ; z\right)$ by

$$
\phi\left(\alpha_{1}, \ldots, \alpha_{q} ; \beta_{1}, \ldots, \beta_{s} ; z\right)=\frac{1}{z}+\sum_{k=0}^{\infty} \frac{\left(\alpha_{1}\right)_{k+1} \ldots\left(\alpha_{q}\right)_{k+1}}{\left(\beta_{1}\right)_{k+1} \ldots\left(\beta_{s}\right)_{k+1}(k+1) !} z^{k},
$$

$\left(q \leq s+1 ; s \in \mathbb{N}_{0}=\mathbb{N} \cup\{0\} ; \mathbb{N}=\{1,2, \ldots\} ; z \in E\right)$,

where $(v)_{k}$ is the Pochhammer symbol (or shifted factorial) defined in (terms of the Gamma function) by

$$
(v)_{k}=\frac{\Gamma(v+k)}{\Gamma(v)}= \begin{cases}1 & \text { if } k=0 \text { and } v \in \mathbb{C} \backslash\{0\} \\ v(v+1) \ldots(v+k-1) & \text { if } k \in \mathbb{N} \text { and } v \in \mathbb{C} .\end{cases}
$$

Now we introduce the following operator

$$
I_{\mu}^{p}\left(\alpha_{1}, \ldots, \alpha_{q}, \beta_{1}, \ldots, \beta_{s}\right): \mathcal{M} \longrightarrow \mathcal{M}
$$


as follows:

Let $F_{\mu, p}(z)=\frac{1}{z}+\sum_{k=0}^{\infty}\left(\frac{k+\mu+1}{\mu}\right)^{p} z^{k}, p \in \mathbb{N}_{0}, \mu \neq 0$ and let $F_{\mu, p}^{-1}(z)$ be defined such that

$$
F_{\mu, p}(z) * F_{\mu, p}^{-1}(z)=\phi\left(\alpha_{1}, \ldots, \alpha_{q} ; \beta_{1}, \ldots, \beta_{s} ; z\right) .
$$

Then

$$
I_{\mu}^{p}\left(\alpha_{1}, \ldots, \alpha_{q}, \beta_{1}, \ldots, \beta_{s}\right) f(z)=F_{\mu, p}^{-1}(z) * f(z) .
$$

From (1.6) it can be easily seen

$$
\begin{aligned}
& I_{\mu}^{p}\left(\alpha_{1}, \ldots \alpha_{q}, \beta_{1}, \ldots \beta_{s}\right) f(z) \\
& =\frac{1}{z}+\sum_{k=0}^{\infty}\left(\frac{\mu}{k+\mu+1}\right)^{p} \frac{\left(\alpha_{1}\right)_{k+1} \ldots\left(\alpha_{q}\right)_{k+1}}{\left(\beta_{1}\right)_{k+1} \ldots\left(\beta_{s}\right)_{k+1}(k+1) !} a_{k} z^{k} .
\end{aligned}
$$

For conveniences, we shall henceforth denote

$$
I_{\mu}^{p}\left(\alpha_{1}, \ldots \alpha_{q}, \beta_{1}, \ldots \beta_{s}\right) f(z)=I_{\mu}^{p}\left(\alpha_{1}, \beta_{1}\right) f(z) .
$$

For the choices of the parameters $p=0, q=2, s=1$, the operator $I_{\mu}^{p}\left(\alpha_{1}, \beta_{1}\right) f(z)$ is reduced to an operator by N. E. Cho and K. I. Noor [2] and K. I. Noor [9] and when $p=0, q=2, s=1, \alpha_{1}=\lambda, \alpha_{2}=1, \beta_{1}=(n+1)$, the operator $I_{\mu}^{p}\left(\alpha_{1}, \beta_{1}\right) f(z)$ is reduced to an operator recently introduced by S.-M. Yuan et al. in [20].

It can be easily verified from the above definition of the operator $I_{\mu}^{p}\left(\alpha_{1}, \beta_{1}\right)$ that

$$
z\left(I_{\mu}^{p+1}\left(\alpha_{1}, \beta_{1}\right) f(z)\right)^{\prime}=\mu I_{\mu}^{p}\left(\alpha_{1}, \beta_{1}\right) f(z)-(\mu+1) I_{\mu}^{p+1}\left(\alpha_{1}, \beta_{1}\right) f(z)
$$

and

$$
z\left(I_{\mu}^{p}\left(\alpha_{1}, \beta_{1}\right) f(z)\right)^{\prime}=\alpha_{1} I_{\mu}^{p}\left(\alpha_{1}+1, \beta_{1}\right) f(z)-\left(\alpha_{1}+1\right) I_{\mu}^{p}\left(\alpha_{1}, \beta_{1}\right) f(z)
$$

By using the operator $I_{\mu}^{p}\left(\alpha_{1}, \beta_{1}\right)$, we now introduce the following subclasses of meromorphic functions:

Definition $1.1([9])$. A function $f \in \mathcal{M}$ is said to belong to the class $M R_{k}(\gamma)$ for $z \in E^{*}, 0 \leq \gamma<1, k \geq 2$, if and only if

$$
-\frac{z f^{\prime}(z)}{f(z)} \in P_{k}(\gamma),
$$

and $f \in M V_{k}(\gamma)$, for $z \in E^{*}, 0 \leq \gamma<1, k \geq 2$, if and only if

$$
-\frac{(z f(z))^{\prime}}{f^{\prime}(z)} \in P_{k}(\gamma) \text {. }
$$

We call $f \in M R_{k}(\gamma)$ a meromorphic function with bounded radius rotation of order $\gamma$ and $f \in M V_{k}(\gamma)$ a meromorphic function with bounded boundary rotation. 
Definition 1.2. Let $f \in \mathcal{M}, 0 \leq \gamma<1, k \geq 2, z \in E^{*}$. Then

$$
f \in M R_{k, \mu}^{p}\left(\alpha_{1}, \beta_{1}, \gamma\right) \text { if and only if } I_{\mu}^{p}\left(\alpha_{1}, \beta_{1}\right) f \in M R_{k}(\gamma) \text {. }
$$

Also

$f \in M V_{k, \mu}^{p}\left(\alpha_{1}, \beta_{1}, \gamma\right)$ if and only if $I_{\mu}^{p}\left(\alpha_{1}, \beta_{1}\right) f \in M V_{k}(\gamma), \quad z \in E^{*}$.

We observe that, for $z \in E^{*}$,

$$
f \in M V_{k, \mu}^{p}\left(\alpha_{1}, \beta_{1}, \gamma\right) \Leftrightarrow-z f^{\prime} \in M R_{k, \mu}^{p}\left(\alpha_{1}, \beta_{1}, \gamma\right) .
$$

Definition 1.3. Let $\lambda \geq 0, f \in \mathcal{M}, p \in \mathbb{N}_{0}, 0 \leq \gamma, \rho<1, \mu>0$ and $z \in E^{*}$. Then $f \in B_{k, \mu}^{\lambda, p}\left(\alpha_{1}, \beta_{1}, \gamma, \rho\right)$, if and only if there exists a function $g \in M V_{2, \mu}^{p}\left(\alpha_{1}, \beta_{1}, \gamma\right)$, such that

$$
\left\{(1-\lambda) \frac{\left(I_{\mu}^{p}\left(\alpha_{1}, \beta_{1}\right) f(z)\right)^{\prime}}{\left(I_{\mu}^{p}\left(\alpha_{1}, \beta_{1}\right) g(z)\right)^{\prime}}+\lambda\left[-\frac{\left(z\left(I_{\mu}^{p}\left(\alpha_{1}, \beta_{1}\right) f(z)\right)^{\prime}\right)^{\prime}}{\left(I_{\mu}^{p}\left(\alpha_{1}, \beta_{1}\right) g(z)\right)^{\prime}}\right]\right\} \in P_{k}(\rho) .
$$

In particular, for $\lambda=0=p, k=q=\mu=2$ and $s=1$, we obtain the class of meromorphic close-to-convex function, see [7], see also K. I. Noor [9]. For $\lambda=1, p=0, k=q=\mu=2, s=1$, we have the class of meromorphic quasi-convex functions defined for $z \in E^{*}$. We note that the class $C^{*}$ of quasi-convex univalent functions, analytic in $E$, was first introduced and studied in [12], see also [13,15].

In order to establish our main results, we need the following lemma, which is properly known as the Miller-Mocanu Lemma.

Lemma $1.1([8])$. Let $u=u_{1}+i u_{2}, v=v_{1}+i v_{2}$ and $\Psi(u, v)$ be a complex valued function satisfying the conditions:

(i) $\Psi(u, v)$ is continuous in a domain $D \subset \mathbb{C}^{2}$,

(ii) $(1,0) \in D$ and $\operatorname{Re} \Psi(1,0)>0$,

(iii) $\operatorname{Re} \Psi\left(i u_{2}, v_{1}\right) \leq 0$, whenever $\left(i u_{2}, v_{1}\right) \in D$ and $v_{1} \leq-\frac{1}{2}\left(1+u_{2}^{2}\right)$.

If $h(z)=1+c_{1} z+c_{2} z^{2}+\cdots$ is a function analytic in $E$ such that $\left(h(z), z h^{\prime}(z)\right) \in D$ and $\operatorname{Re}\left(\Psi\left(h(z), z h^{\prime}(z)\right)>0\right.$ for $z \in E$, then $\operatorname{Re} h(z)>0$ in $E$.

\section{Main results.}

Theorem 2.1. Let $\operatorname{Re} \alpha_{1}>0, \mu>0$ and $0 \leq \gamma<1$. Then

$$
M R_{k, \mu}^{p}\left(\alpha_{1}+1, \beta_{1}, \gamma\right) \subset M R_{k, \mu}^{p}\left(\alpha_{1}, \beta_{1}, \rho\right) \subset M R_{k, \mu}^{p+1}\left(\alpha_{1}, \beta_{1}, \eta\right) .
$$

Proof. We prove the first part of the Theorem 2.1 and the second part follows by using similar techniques. Let

$$
f \in M R_{k, \mu}^{p}\left(\alpha_{1}+1, \beta_{1}, \gamma\right), \quad z \in E^{*}
$$

and set

$$
-\frac{\left(I_{\mu}^{p}\left(\alpha_{1}, \beta_{1}\right) f(z)\right)^{\prime}}{\left(I_{\mu}^{p}\left(\alpha_{1}, \beta_{1}\right) f(z)\right)}=\left(\frac{k}{4}+\frac{1}{2}\right) h_{1}(z)-\left(\frac{k}{4}-\frac{1}{2}\right) h_{2}(z)=H(z) .
$$


Simple computation together with (2.1) and (1.10) yields

$$
-\frac{\left(I_{\mu}^{p}\left(\alpha_{1}+1, \beta_{1}\right) f(z)\right)^{\prime}}{\left(I_{\mu}^{p}\left(\alpha_{1}+1, \beta_{1}\right) f(z)\right)}=\left[H(z)+\frac{z H^{\prime}(z)}{-H(z)+\alpha_{1}+1}\right] \in P_{k}(\gamma), z \in E .
$$

Let

then

$$
\Phi_{\alpha_{1}}(z)=\frac{1}{\alpha_{1}+1}\left[\frac{1}{z}+\sum_{k=0}^{\infty} z^{k}\right]+\frac{\alpha_{1}}{\alpha_{1}+1}\left[\frac{1}{z}+\sum_{k=0}^{\infty} k z^{k}\right],
$$

$$
\begin{aligned}
H(z) * z \Phi_{\alpha_{1}}(z)= & {\left[H(z)+\frac{z H^{\prime}(z)}{-H(z)+\alpha_{1}+1}\right] } \\
= & \left(\frac{k}{4}+\frac{1}{2}\right)\left[h_{1}(z)+\frac{z h_{1}^{\prime}(z)}{-h_{1}(z)+\alpha_{1}+1}\right] \\
& -\left(\frac{k}{4}-\frac{1}{2}\right)\left[h_{2}(z)+\frac{z h_{2}^{\prime}(z)}{-h_{2}(z)+\alpha_{1}+1}\right] .
\end{aligned}
$$

Since $f \in M R_{k, \mu}^{p}\left(\alpha_{1}+1, \beta_{1}, \gamma\right)$, it follows from (2.2) and (2.3) that

$$
\left[h_{i}(z)+\frac{z h_{i}^{\prime}(z)}{-h_{i}(z)+\alpha_{1}+1}\right] \in P(\gamma), \quad i=1,2, \quad z \in E .
$$

Let $h_{i}(z)=(1-\rho) p_{i}(z)+\rho$. Then

$$
\left\{(1-\rho) p_{i}(z)+\rho-\gamma+\frac{(1-\rho) z p_{i}^{\prime}(z)}{-(1-\rho) p_{i}(z)-\rho+\alpha_{1}+1}\right\} \in P, \quad z \in E .
$$

We shall show that $p_{i}(z) \in P, i=1,2$.

We form the functional $\Psi(u, v)$ by taking $u=u_{1}+i u_{2}=p_{i}(z), v=$ $v_{1}+i v_{2}=z p_{i}^{\prime}(z)$. The first two conditions of Lemma 1.1 can be easily verified. We need to verify condition $(i i i)$ as follows:

$$
\Psi(u, v)=\left\{(1-\rho) u+\rho-\gamma+\frac{(1-\rho) v}{-(1-\rho) u-\rho+\alpha_{1}+1}\right\},
$$

implies that

$$
\operatorname{Re} \Psi\left(i u_{2}, v_{1}\right)=\rho-\gamma+\frac{(1-\rho)\left(\alpha_{1}+1-\rho\right) v_{1}}{(1-\rho)^{2} u_{2}^{2}+\left(-\rho+\alpha_{1}+1\right)^{2}} .
$$

By taking $v_{1} \leq-\frac{1}{2}\left(1+u_{2}^{2}\right)$, we have

$$
\operatorname{Re} \Psi\left(i u_{2}, v_{1}\right) \leq \frac{A+B u_{2}^{2}}{2 C},
$$

where

$$
\begin{aligned}
& A=2(\rho-\gamma)\left(\alpha_{1}+1-\rho\right)^{2}-(1-\rho)\left(\alpha_{1}+1-\rho\right), \\
& B=2(\rho-\gamma)(1-\rho)^{2}-(1-\rho)\left(\alpha_{1}+1-\rho\right), \\
& C=\left(\alpha_{1}+1-\rho\right)^{2}+(1-\rho)^{2} u_{2}^{2}>0 .
\end{aligned}
$$


We note that $\operatorname{Re} \Psi\left(i u_{2}, v_{1}\right) \leq 0$ if and only if $A \leq 0$ and $B \leq 0$. From $A \leq 0$, we obtain

$$
\rho=\frac{1}{4}\left\{\left(3+2 \alpha_{1}+2 \gamma\right)-\sqrt{\left(3+2 \alpha_{1}+2 \gamma\right)^{2}-8}\right\},
$$

and $B \leq 0$ gives us $0 \leq \rho<1$.

Now using Lemma 1.1, we see that $p_{i}(z) \in P$ for $z \in E, i=1,2$ and hence $f \in M R_{k, \mu}^{p}\left(\alpha_{1}, \beta_{1}, \rho\right)$ with $\rho$ given by (2.4).

In particular, we note that

$$
\rho=\frac{1}{4}\left\{\left(3+2 \alpha_{1}\right)-\sqrt{\left(12 \alpha_{1}+4 \alpha_{1}^{2}\right)+1}\right\} .
$$

Theorem 2.2. Let $\operatorname{Re} \alpha_{1}, \mu>0$. Then

$$
M V_{k, \mu}^{p}\left(\alpha_{1}+1, \beta_{1}, \gamma\right) \subset M V_{k, \mu}^{p}\left(\alpha_{1}, \beta_{1}, \rho\right) \subset M V_{k, \mu}^{p+1}\left(\alpha_{1}, \beta_{1}, \eta\right) .
$$

Proof. We observe that

$$
\begin{aligned}
f(z) & \in M V_{k, \mu}^{p}\left(\alpha_{1}+1, \beta_{1}, \gamma\right) \Leftrightarrow-z f^{\prime}(z) \in M R_{k, \mu}^{p}\left(\alpha_{1}+1, \beta_{1}, \gamma\right) \\
& \Rightarrow-z f^{\prime}(z) \in M R_{k, \mu}^{p}\left(\alpha_{1}, \beta_{1}, \rho\right) \\
& \Leftrightarrow f(z) \in M V_{k, \mu}^{p}\left(\alpha_{1}, \beta_{1}, \rho\right),
\end{aligned}
$$

where $\rho$ is given by $(2.4)$.

The second part can be proved by means of similar arguments.

Theorem 2.3. Let $\operatorname{Re} \alpha_{1}, \mu>0$. Then

$$
B_{k, \mu}^{\lambda, p}\left(\alpha_{1}+1, \beta_{1}, \gamma_{1}, \rho_{1}\right) \subset B_{k, \mu}^{\lambda, p}\left(\alpha_{1}, \beta_{1}, \gamma_{2}, \rho_{2}\right) \subset B_{k, \mu}^{\lambda, p+1}\left(\alpha_{1}, \beta_{1}, \gamma_{3}, \rho_{3}\right),
$$

where $\gamma_{i}=\gamma_{i}\left(\rho_{i}, \mu\right), i=1,2,3$ are given in the proof.

Proof. We prove the first inclusion of this result and the other part follows along similar lines.

Let $f \in B_{k, \mu}^{\lambda, p}\left(\alpha_{1}+1, \beta_{1}, \gamma_{1}, \rho_{1}\right)$. Then by Definition 1.3 , there exists a function $g \in M V_{2, \mu}^{p}\left(\alpha_{1}+1, \beta_{1}, \gamma_{1}\right)$ such that

$$
\begin{aligned}
\{(1-\lambda) & \frac{\left(I_{\mu}^{p}\left(\alpha_{1}+1, \beta_{1}\right) f(z)\right)^{\prime}}{\left(I_{\mu}^{p}\left(\alpha_{1}+1, \beta_{1}\right) g(z)\right)^{\prime}} \\
& \left.+\lambda\left[-\frac{\left(z\left(I_{\mu}^{p}\left(\alpha_{1}+1, \beta_{1}\right) f(z)\right)^{\prime}\right)^{\prime}}{\left(I_{\mu}^{p}\left(\alpha_{1}+1, \beta_{1}\right) g(z)\right)^{\prime}}\right]\right\} \in P_{k}\left(\rho_{1}\right) .
\end{aligned}
$$

Set

$$
h(z)=\left\{(1-\lambda) \frac{\left(I_{\mu}^{p}\left(\alpha_{1}, \beta_{1}\right) f(z)\right)^{\prime}}{\left(I_{\mu}^{p}\left(\alpha_{1}, \beta_{1}\right) g(z)\right)^{\prime}}+\lambda\left[-\frac{\left(z\left(I_{\mu}^{p}\left(\alpha_{1}, \beta_{1}\right) f(z)\right)^{\prime}\right)^{\prime}}{\left(I_{\mu}^{p}\left(\alpha_{1}, \beta_{1}\right) g(z)\right)^{\prime}}\right]\right\},
$$

where $h(z)$ is an analytic function in $E$ with $h(0)=1$. 
Now, $g \in M V_{2, \mu}^{p}\left(\alpha_{1}+1, \beta_{1}, \gamma_{1}\right) \subset M V_{2, \mu}^{p}\left(\alpha_{1}, \beta_{1}, \gamma_{2}\right)$, where $\gamma_{2}$ is given by the equation

$$
2 \gamma_{2}^{2}+\left(3+2 \alpha_{1}-2 \gamma_{1}\right) \gamma_{2}-\left\{2 \gamma_{1}\left(1+\alpha_{1}\right)+1\right\}=0
$$

Therefore,

$$
q(z)=-\frac{\left(z I_{\mu}^{p}\left(\alpha_{1}, \beta_{1}\right) g(z)\right)^{\prime}}{\left(I_{\mu}^{p}\left(\alpha_{1}, \beta_{1}\right) g(z)\right)^{\prime}} \in P\left(\gamma_{2}\right), \quad z \in E .
$$

By using (1.10), (2.5), (2.6) and (2.7), we have

$$
\left\{h(z)+\frac{\lambda z h^{\prime}(z)}{-q(z)+\alpha_{1}+1}\right\} \in P_{k}\left(\rho_{1}\right), q(z) \in P\left(\gamma_{2}\right), \quad z \in E .
$$

With

$$
h(z)=\left(\frac{k}{4}+\frac{1}{2}\right)\left[\left(1-\rho_{2}\right) h_{1}(z)+\rho_{2}\right]-\left(\frac{k}{4}-\frac{1}{2}\right)\left[\left(1-\rho_{2}\right) h_{2}(z)+\rho_{2}\right],
$$

(2.8) can be written as

$$
\begin{aligned}
& \left(\frac{k}{4}+\frac{1}{2}\right)\left\{\left(1-\rho_{2}\right) h_{1}(z)+\rho_{2}+\frac{\left(1-\rho_{2}\right) \lambda z h_{1}^{\prime}(z)}{-q(z)+\alpha_{1}+1}\right\} \\
& -\left(\frac{k}{4}-\frac{1}{2}\right)\left\{\left(1-\rho_{2}\right) h_{2}(z)+\rho_{2}+\frac{\left(1-\rho_{2}\right) \lambda z h_{2}^{\prime}(z)}{-q(z)+\alpha_{1}+1}\right\},
\end{aligned}
$$

where

$$
\left\{\left(1-\rho_{2}\right) h_{i}(z)+\rho_{2}+\frac{\left(1-\rho_{2}\right) \lambda z h_{i}^{\prime}(z)}{-q(z)+\alpha_{1}+1}\right\} \in P\left(\rho_{1}\right), z \in E, i=1,2 .
$$

That is

$$
\left\{\left(1-\rho_{2}\right) h_{i}(z)+\rho_{2}-\rho_{1}+\frac{\left(1-\rho_{2}\right) \lambda z h_{i}^{\prime}(z)}{-q(z)+\alpha_{1}+1}\right\} \in P, z \in E, i=1,2 .
$$

We form the functional $\Psi(u, v)$ by choosing $u=u_{1}+i u_{2}=h_{i}(z), v=$ $v_{1}+i v_{2}=z h_{i}^{\prime}(z)$, and

$$
\Psi(u, v)=\left\{\left(1-\rho_{2}\right) u+\rho_{2}-\rho_{1}+\frac{\left(1-\rho_{2}\right) \lambda v}{-q(z)+\alpha_{1}+1}\right\}, \quad\left(q=q_{1}+i q_{2}\right) .
$$

The first two conditions of Lemma 1.1 are clearly satisfied. We verify $($ iii $)$, with $v_{1} \leq-\frac{1}{2}\left(1+u_{2}^{2}\right)$ as follows:

$$
\begin{aligned}
& \operatorname{Re} \Psi\left(i u_{2}, v_{1}\right)=\rho_{2}-\rho_{1}+\operatorname{Re}\left\{\frac{\lambda\left(1-\rho_{2}\right) v_{1}\left\{\left(-q_{1}+\alpha_{1}+1\right)+i q_{2}\right\}}{\left(-q_{1}+\alpha_{1}+1\right)^{2}+q_{2}^{2}}\right\} \\
& \leq \frac{2\left(\rho_{2}-\rho_{1}\right)\left|-q+\alpha_{1}+1\right|^{2}-\lambda\left(1-\rho_{2}\right)\left\{\left(-q_{1}+\alpha_{1}+1\right)\left(1+u_{2}^{2}\right)\right.}{2\left|-q+\alpha_{1}+1\right|^{2}} \\
& =\frac{A+B u_{2}^{2}}{2 C} \leq 0,
\end{aligned}
$$


if $A \leq 0$ and $B \leq 0$, where

$$
\begin{aligned}
& A=2\left(\rho_{2}-\rho_{1}\right)\left|-q+\alpha_{1}+1\right|^{2}-\lambda\left(1-\rho_{2}\right)\left\{\left(-q_{1}+\alpha_{1}+1\right),\right. \\
& B=-\lambda\left(1-\rho_{2}\right)\left\{\left(-q_{1}+\alpha_{1}+1\right) \leq 0,\right. \\
& C=\left|-q+\alpha_{1}+1\right|^{2}>0 .
\end{aligned}
$$

From $A \leq 0$, we obtain

$$
\rho_{2}=\frac{2 \rho_{1}\left|-q+\alpha_{1}+1\right|^{2}+\lambda \operatorname{Re}\left(-q(z)+\alpha_{1}+1\right)}{2\left|-q+\alpha_{1}+1\right|^{2}+\lambda \operatorname{Re}\left(-q(z)+\alpha_{1}+1\right)} .
$$

Hence, using Lemma 1.1, it follows that $h(z)$, defined by (2.6), belongs to $P_{k}\left(\rho_{2}\right)$ and thus $f \in B_{k, \mu}^{\lambda, p}\left(\alpha_{1}, \beta_{1}, \gamma_{2}, \rho_{2}\right)$ for $z \in E^{*}$. This completes the proof of the first part. The second part of this result can be obtained by using similar techniques and the relation (1.9).

Theorem 2.4. Let $\operatorname{Re} \alpha_{1}, \mu>0$. Then

(i) $B_{k, \mu}^{\lambda, p}\left(\alpha_{1}, \beta_{1}, \gamma, \rho\right) \subset B_{k, \mu}^{0, p}\left(\alpha_{1}, \beta_{1}, \gamma, \rho_{4}\right)$.

(ii) $B_{k, \mu}^{\lambda_{1}, p}\left(\alpha_{1}, \beta_{1}, \gamma, \rho\right) \subset B_{k, \mu}^{\lambda_{2}, p}\left(\alpha_{1}, \beta_{1}, \gamma, \rho\right)$, for $0 \leq \lambda_{2}<\lambda_{1}$.

Proof. $(i)$. Let

$$
h(z)=\frac{\left(I_{\mu}^{p}\left(\alpha_{1}, \beta_{1}\right) f(z)\right)^{\prime}}{\left(I_{\mu}^{p}\left(\alpha_{1}, \beta_{1}\right) g(z)\right)^{\prime}},
$$

$h(z)$ is analytic in $E$ and $h(0)=1$. Then

$$
\begin{aligned}
\{(1-\lambda) & \left.\frac{\left(I_{\mu}^{p}\left(\alpha_{1}, \beta_{1}\right) f(z)\right)^{\prime}}{\left(I_{\mu}^{p}\left(\alpha_{1}, \beta_{1}\right) g(z)\right)^{\prime}}+\lambda\left[-\frac{\left(z\left(I_{\mu}^{p}\left(\alpha_{1}, \beta_{1}\right) f(z)\right)^{\prime}\right)^{\prime}}{\left(I_{\mu}^{p}\left(\alpha_{1}, \beta_{1}\right) g(z)\right)^{\prime}}\right]\right\} \\
& =h(z)+\lambda \frac{z h^{\prime}(z)}{-h_{0}(z)},
\end{aligned}
$$

where

$$
h_{0}(z)=-\frac{\left(z\left(I_{\mu}^{p}\left(\alpha_{1}, \beta_{1}\right) f(z)\right)^{\prime}\right)^{\prime}}{\left(I_{\mu}^{p}\left(\alpha_{1}, \beta_{1}\right) g(z)\right)^{\prime}} \in P(\gamma) .
$$

Since $f \in B_{k, \mu}^{\lambda, p}\left(\alpha_{1}, \beta_{1}, \gamma, \rho\right)$, it follows that

$$
\left[h(z)+\lambda \frac{z h^{\prime}(z)}{-h_{0}(z)}\right] \in P_{k}(\rho), h_{0} \in P(\gamma) \text {, for } z \in E .
$$

Let

$$
h(z)=\left(\frac{k}{4}+\frac{1}{2}\right) h_{1}(z)-\left(\frac{k}{4}-\frac{1}{2}\right) h_{2}(z) .
$$

Thus (2.10) implies that

$$
\left[h_{i}(z)+\lambda \frac{z h_{i}^{\prime}(z)}{-h_{0}(z)}\right] \in P(\rho), \quad z \in E, i=1,2 .
$$


and using similar techniques, together with Lemma 1.1, it follows that $h_{i}(z) \in P\left(\rho_{4}\right), i=1,2$, where

$$
\rho_{4}=\frac{2 \rho\left|h_{0}(z)\right|^{2}+\lambda \operatorname{Re} h_{0}(z)}{2\left|h_{0}(z)\right|^{2}+\lambda \operatorname{Re} h_{0}(z)} .
$$

Therefore $h(z) \in P_{k}\left(\rho_{4}\right)$, and $f \in B_{k, \mu}^{0, p}\left(\alpha_{1}, \beta_{1}, \gamma, \rho_{4}\right)$, for $z \in E^{*}$. In particular, it can be shown that $h_{i}(z) \in P(\rho), i=1,2$. Consequently $h \in P_{k}(\rho)$ and $f \in B_{k, \mu}^{0, p}\left(\alpha_{1}, \beta_{1}, \gamma, \rho\right)$ in $E^{*}$.

For $\lambda_{2}=0$, we have part $(i)$. Therefore, we let $\lambda_{2}>0$ and $f \in$ $B_{k, \mu}^{\lambda_{1}, p}\left(\alpha_{1}, \beta_{1}, \gamma, \rho\right)$. There exist two functions $H_{1}(z), H_{2}(z) \in P_{k}(\rho)$ such that

$$
\begin{gathered}
\left\{\left(1-\lambda_{1}\right) \frac{\left(I_{\mu}^{p}\left(\alpha_{1}+1, \beta_{1}\right) f(z)\right)^{\prime}}{\left(I_{\mu}^{p}\left(\alpha_{1}+1, \beta_{1}\right) g(z)\right)^{\prime}}+\lambda_{1}\left[-\frac{\left(z\left(I_{\mu}^{p}\left(\alpha_{1}+1, \beta_{1}\right) f(z)\right)^{\prime}\right)^{\prime}}{\left(I_{\mu}^{p}\left(\alpha_{1}+1, \beta_{1}\right) g(z)\right)^{\prime}}\right]\right\}=H_{1}(z) \\
\frac{\left(I_{\mu}^{p}\left(\alpha_{1}+1, \beta_{1}\right) f(z)\right)^{\prime}}{\left(I_{\mu}^{p}\left(\alpha_{1}+1, \beta_{1}\right) g(z)\right)^{\prime}}=H_{2}(z),
\end{gathered}
$$

where $g(z) \in M V_{2, \mu}^{p}\left(\alpha_{1}, \beta_{1}, \gamma\right)$.

Now

$$
\begin{aligned}
& \left\{\left(1-\lambda_{2}\right) \frac{\left(I_{\mu}^{p}\left(\alpha_{1}+1, \beta_{1}\right) f(z)\right)^{\prime}}{\left(I_{\mu}^{p}\left(\alpha_{1}+1, \beta_{1}\right) g(z)\right)^{\prime}}+\lambda_{2}\left[-\frac{\left(z\left(I_{\mu}^{p}\left(\alpha_{1}+1, \beta_{1}\right) f(z)\right)^{\prime}\right)^{\prime}}{\left(I_{\mu}^{p}\left(\alpha_{1}+1, \beta_{1}\right) g(z)\right)^{\prime}}\right]\right\} \\
& =\frac{\lambda_{2}}{\lambda_{1}} H_{1}(z)+\left(1-\frac{\lambda_{2}}{\lambda_{1}}\right) H_{2}(z) .
\end{aligned}
$$

Since the class $P_{k}(\rho)$ is convex, see [10], it follows that the right hand side of (2.11) belongs to $P_{k}(\rho)$ and this shows that $f \in B_{k, \mu}^{\lambda_{2}, p}\left(\alpha_{1}, \beta_{1}, \gamma, \rho\right)$ for $z \in E^{*}$. This completes the proof.

Inclusion properties involving the integral operator $\boldsymbol{F}_{\boldsymbol{c}}$. Consider the operator $F_{c}$, defined by

$$
F_{c}(f)(z)=\frac{c}{z^{c+1}} \int_{0}^{z} t^{c} f(t) d t \quad(f \in \mathcal{M} ; c>0) .
$$

From the Definition of $F_{c}$ defined by (2.12), we observe that

(2.13) $z\left(\left(I_{\mu}^{p}\left(\alpha_{1}, \beta_{1}\right) F_{c} f(z)\right)^{\prime}=c\left(I_{\mu}^{p}\left(\alpha_{1}, \beta_{1}\right) f(z)-(c+1)\left(I_{\mu}^{p}\left(\alpha_{1}, \beta_{1}\right) F_{c} f(z)\right.\right.\right.$.

Using (2.12), (2.13) with similar arguments as used earlier, we can prove the following theorem.

Theorem 2.5. Let $f \in M R_{k, \mu}^{p}\left(\alpha_{1}, \beta_{1}, \gamma\right)$ or $f \in M V_{k, \mu}^{p}\left(\alpha_{1}, \beta_{1}, \gamma\right)$ or $f \in$ $B_{k, \mu}^{\lambda, p}\left(\alpha_{1}, \beta_{1}, \gamma, \rho\right)$, for $z \in E$. Then $F_{c}(f)$ defined by (2.12) is also in the same class for $z \in E^{*}$. 
Acknowledgement. I am thankful for the valuable suggestions of referee which improved this paper.

\section{REFERENCES}

[1] Carlson, B. C., Shaeffer, B. D., Starlike and prestarlike hypergeometric functions, SIAM J. Math. Anal. 15 (1984), no. 4, 737-745.

[2] Cho, N. E., Noor, K. I., Inclusion properties for certain classes of meromorphic functions associated with Choi-Saigo-Srivastava operator, J. Math. Anal. Appl. 320 (2006), no. 2, 779-786.

[3] Cho, N. E., Kwon, O. S. and Srivastava, H. M., Inclusion relationships for certain subclasses of meromorphic functions associated with a family of multiflier transformations, Integral Transforms Spec. Funct. 16 (2005), no. 8, 647-659.

[4] Dziok, J., Srivastava, H. M., Classes of analytic functions associated with the generalized hypergeometric function, Appl. Math. Comput. 103 (1999), no. 1, 1-13.

[5] Hohlov, E. Y., Operators and operations in the class of univalent functions, Izv. Vyssh. Uchebn. Zaved. Mat., (1978), no. 10 (197), 83-89 (in Russian).

[6] Jung, I. B., Kim, Y. C. and Srivastava, H. M., The Hardy space of analytic functions associated with certain one-parameter families of integral operators, J. Math. Anal. Appl. 176 (1993), no. 1, 138-147.

[7] Kumar, V., Shukla, S. L., Certain integrals for classes of p-valent meromorphic functions, Bull. Austral. Math. Soc. 25 (1982), no. 1, 85-97.

[8] Miller, S. S., Differential inequalities and Carathéodory functions, Bull. Amer. Math. Soc. 81 (1975), 79-81.

[9] Noor, K. I., On certain classes of meromorphic functions involving integral operators, JIPAM. J. Inequal. Pure Appl. Math. 7 (2006), no. 4, Article 138, 8 pp. (electronic).

[10] Noor, K. I., On subclasses of close-to-convex functions of higher order, Internat. J. Math. Math. Sci. 15 (1992), no. 2, 279-290.

[11] Noor, K. I., On new classes of integral operators, J. Nat. Geom. 16 (1999), no. 1-2, $71-80$.

[12] Noor, K. I., On close-to-convex and related functions, Ph. D. Thesis, University of Wales, Swansea, U. K., 1972.

[13] Noor, K. I., On quasiconvex functions and related topics, Internat. J. Math. Math. Sci. 10 (1987), no. 2, 241-258.

[14] Noor, K. I., Noor, M. A., On integral operators, J. Math. Anal. Appl. 238 (1999), no. $2,341-352$.

[15] Noor, K. I., Thomas, D. K., Quasiconvex univalent functions, Internat. J. Math. Math. Sci. 3 (1980), no. 2, 255-266.

[16] Padmanabhan, K., Parvatham, R., Properties of a class of functions with bounded boundary rotation, Ann. Polon. Math. 31 (1975/76), no. 3, 311-323.

[17] Pinchuk, B., Functions of bounded boundary rotation, Israel J. Math. 10 (1971), 6-16.

[18] Ruscheweyh, S., New criteria for univalent functions, Proc. Amer. Math. Soc. 49 (1975), 109-115.

[19] Selvaraj, C., Karthikeyan, K. R., Some inclusion relationships for certain subclasses of meromorphic functions associated with a family of integral operators, Acta Math. Univ. Comenian. (N. S.) 78 (2009), no. 2, 245-254.

[20] Yuan, S.-M., Liu, Z.-M. and Srivastava, H. M., Some inclusion relationships and integral-preserving properties of certain subclasses of meromorphic functions associated with a family of integral operators, J. Math. Anal. Appl. 337 (2008), no.1, 505-515. 


\author{
Ali Muhammad \\ Department of Basic Sciences \\ University of Engineering and Technology \\ Peshawar \\ Pakistan \\ e-mail: ali7887@gmail.com
}

Received March 25, 2011 